\title{
Association between superior semicircular canal dehiscence and other dehiscences in temporal bone
}

\author{
J. Whyte ${ }^{1}$, A.I. Cisneros ${ }^{1}$, A. Garcia-Barrios ${ }^{1}$, J. Fraile², A. Whyte ${ }^{3}$, R. Crovetto ${ }^{4}$, M. Lahoz ${ }^{1}$ \\ ${ }^{1}$ Anatomy and Histology Department, School of Medicine, Zaragoza University, Spain \\ 2Pathology Department, Miguel Servet Hospital, Spain \\ ${ }^{3}$ Pathology Department, School of Veterinary, Zaragoza University, Spain \\ ${ }^{4}$ Bilbao Clinic, Spain \\ [Received: 14 October 2019; Accepted: 3 December 2019]
}

\begin{abstract}
Background: The study of the association between superior semicircular canal and other dehiscences in the temporal bone.

Materials and methods: We have studied computed tomography of radiologically diagnosed people with superior or posterior semicircular canal dehiscences, in four health centres. In addition, we have studied one isolated human temporal bone, one skull and one cadaver head belonging to the collection of the Department of Human Anatomy and Histology of the University of Zaragoza that had dehiscence in the superior semicircular canal.

Results: The most frequent association that we observed was between superior semicircular canal dehiscence and tegmen tympani dehiscence (37.33\%). Three cases (two clinical cases and one isolated temporal bone) showed multiple associated dehiscences (tegmen tympani, mastoid antrum, posterior semicircular canal, internal auditory canal, glenoid cavity, tympanum bone and geniculate ganglion) associated with superior semicircular canal dehiscence

Conclusions: When the superior semicircular canal dehiscence is associated to other in the petrous bone (tegmen tympani, mastoid antrum, posterior semicircular canal, internal auditory canal) could be grouped into the same syndrome called "otic capsule syndrome", since they have the same origin and common aetiology (otic capsule). (Folia Morphol 2020; 79, 4: 823-828)
\end{abstract}

Key words: bony labyrinth, otic capsule, bony defects group, third window

\section{INTRODUCTION}

A milestone in the study of superior semicircular canal (SSC) pathology was the manuscript by Minor [22] in 1998, who first described the presence of dehiscence in the canal. This dehiscence is a rare and uncommon peripheral vestibulopathy, which is characterised by a lack of bone coverage of the SSC in the area closest to the dura of the middle cerebral fossa. Since that date, studies have been conducted to investigate its prevalence, pathophysiology, clinical variables, diagnosis and surgical procedures.

There are currently two theories about the genesis of dehiscence: congenital (stop or delay in the ossification of the canal during its development) and acquired (bone layer rupture covering the canal as

Address for correspondence: Dr. A.I. Cisneros, Anatomy and Histology Department, School of Medicine, Zaragoza University, Spain, e-mail: aicisner@unizar.es 
a result of another event, as increased intracranial pressure, trauma, even after childbirth or coitus).

The association of SSC dehiscence with other ear diseases $[4,9,27]$ and other dehiscences produced in the bone labyrinth is currently being investigated.

In this line, associations between dehiscence of the SSC and dehiscence of the posterior semicircular canal have been described [6,15, 18, 20,21]. Both dehiscences show a similar, nonspecific clinical presentation, sometimes the auditory symptoms (hypoacusis, tinnitus) and sometimes the vestibular ones (dizziness, nystagmus, Tullio phenomenon) predominate; they may not even present any.

There may also be cases where there are dehiscences in other structures such as the tegmen tympani $[1,5,24]$, geniculate ganglion $[10,13,17]$ and internal auditory canal [19].

The objective of this study is to determine radiologically on the one hand, the possible associations between dehiscence in the superior and posterior semicircular canal, and on the other, both dehiscences with other morphological alterations in the temporal bone (glenoid cavity and internal auditory canal).

\section{MATERIALS AND METHODS}

We have studied computed tomography (CT) of radiologically diagnosed people with superior or posterior semicircular canal dehiscences, in four health centres: Basurto and Cruces Hospital, General Hospital of the Defence and Miguel Servet Hospital in Zaragoza. An informed consent form was signed by every participating patient. All the patients acknowledged that they cannot be identified via the paper; the researchers fully anonymised them. The ethics committee of each centre approved this study in accordance with the guidelines of the Helsinki Declaration of 1983.

The studies have been performed with multi-splice helical CT equipment (Philips Brilliance 6), obtaining the images on the axial plane and with the patient's neck in hyperextension to avoid direct radiation damage to crystalline. Later, coronal reconstructions have been carried out in all the cases, in the plane of the SSC of each ear (Pöschl plane) and in axial planes for the posterior semicircular canals. The roof of the glenoid cavity has been studied with sagittal plane reconstructions.

The "raw data" have been reconstructed using a bone algorithm.
Table 1. Distribution of superior semicircular canal (SSC) dehiscence and posterior semicircular canal (PSC) dehiscence

\begin{tabular}{lccc}
\hline & Right side & Left side & Bilateral \\
\hline SSC & $31 / 71(43.67 \%)$ & $20 / 71(28.17 \%)$ & $20 / 71(28.16 \%)$ \\
PSC & $1 / 4(25 \%)$ & $1 / 4(25 \%)$ & $2 / 4(50 \%)$ \\
\hline
\end{tabular}

The following radiological protocols for the acquisition and formatting of the images have been used: $2 \times 0.6 \mathrm{~mm}$ collimation, $0.65 \mathrm{~mm}$ splice thickness, $0.32 \mathrm{~mm}$ splice increase, 0.75 s rotation time, 0.38 pitch, $120 \mathrm{CV}, 300 \mathrm{mAs}, 1024 \times 1024$ matrix, $180 \mathrm{~mm}$ field of vision, $0.5 \mathrm{~mm}$ reconstruction thickness and $0.5 \mathrm{~mm}$ reconstruction increase.

The CT scans were updated by 3 radiologists with more than 10 years of experience in neuroradiology and reviewed by the signatories of the manuscript, all of them with extensive experience in dehiscence of semicircular canals.

\section{RESULTS}

We have performed a radiological study on a total of 61 patients ( 20 men, 41 women) with an average age of 55.98 years (ranging 2-89), in terms of the possible associations between (superior and posterior) vertical semicircular canal dehiscence, and other dehiscences located in the temporal bone. Two of the patients, who presented unilateral SSC dehiscence, were sisters.

Seventy one cases of SSC dehiscence and 4 of posterior semicircular canal dehiscence were detected. Thirty-one dehiscences were detected on the right side in the SSC, 20 on the left side, and bilateral dehiscences $(28.16 \%)$ were observed in 10 patients (20 cases) (Table 1). Two dehiscences were detected on the right side in the posterior semicircular canal, and another two on the left side, and there was one bilateral case.

The most frequent association that we observed was between SSC dehiscence and tegmen tympani dehiscence, which was verified in a total of 28 (39.44\%) cases.

Two cases presented multiple associated dehiscences (Fig. 1). Thus, the first case presented bilateral superior semicircular dehiscences (Fig. 1A), bilateral tegmen tympani dehiscences, and dehiscence in the glenoid cavity of the temporomandibular joint on the 

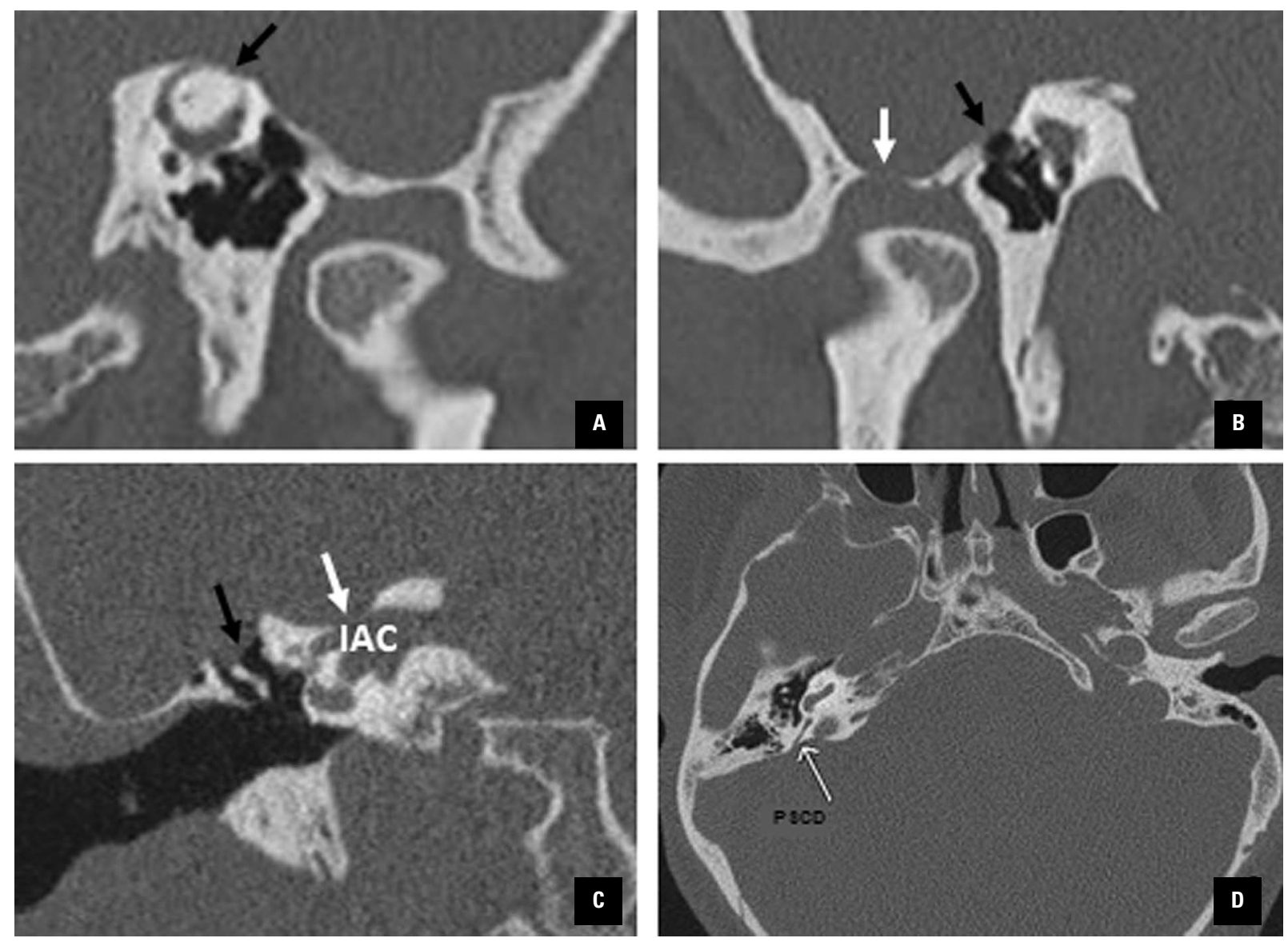

Figure 1. Different associations between superior semicircular canal dehiscence with others in the temporal bone; $\mathbf{A}$. Superior semicircular canal dehiscence (black arrow); B. Tegmen tympani (black arrow), glenoid cavity (white arrow); C. Tegmen tympani (black arrow), internal auditory canal (IAC; white arrow); D. Posterior semicircular canal dehiscence (PSCD; white arrow).

Table 2. Association of temporal bone dehiscences

\begin{tabular}{lccc}
\hline & TTD & IACD & GFD \\
\hline SSCD & $28 / 71(39.44 \%)$ & $1 / 71(1.41 \%)$ & $1 / 71(1.41 \%)$ \\
PSCD & $2 / 4(50 \%)$ & $1 / 4(25 \%)$ & $0 / 4(0 \%)$ \\
\hline
\end{tabular}

SSCD — superior semicircular canal dehiscence; PSCD — posterior semicircular canal dehiscence; TTD — tegmen tympani dehiscence; IACD — internal auditory canal dehiscence; GFD — glenoid cavity dehiscence

right side (Fig. 1B). The other cases showed bilateral superior and posterior semicircular canal dehiscences (Fig. 1D), bilateral tegmen tympani dehiscences, and dehiscence of the roof of the internal auditory canal (Fig. 1C) on the right side (Table 2).

\section{DISCUSSION}

Superior semicircular canal dehiscence, as a structural cause of dizziness and hypoacusis, is only 20 years old [22], so there are still knowledge gaps related to this pathology. Five years later it would be Krombach et al. [14] who described posterior semicircular canal dehiscence.

The first authors who described associations between defects of the bony coverage of the SSC and other parts of the bony labyrinth was Minor [23] who observed an association between tegmen dehiscence and the SSC. One year later, Gianoli [10] underlined the high incidence of patients who also presented radiographic superior canal dehiscence with geniculate ganglion dehiscence. Later, Manzari and Modugno [18] and Chen et al. [3] described isolated cases of SSC dehiscences associated with posterior canal dehiscences, the former in adults, and the latter in children.

In large series of persons with SSC dehiscence, the affectation was found to be bilateral, in percentages that varied between $16 \%$ and $37 \%$. These data are very similar to those observed by us, as we have registered an incidence of $34.48 \%$. 
The presence of bilateral posterior semicircular canal dehiscences is not very frequent, and they are limited to isolated cases such as those described by Saxby et al. [30] and Bear et al. [2]. We have also observed one single isolated case of bilateral dehiscence, but we must consider that posterior semicircular dehiscence is much less frequent than superior semicircular dehiscence, being $3.6 \%$ and $0.6 \%$, respectively [5].

The association between superior and posterior canal dehiscence has been described by Krombach et al. [14], who observed 8 patients with posterior and SSC dehiscences. Chen et al. [3] also obtained 1 case with both dehiscences. Gopen et al. [11] observed that 2 of the 12 patients with posterior semicircular canal dehiscences also presented SSC dehiscences. Russo et al. [29] described that in 3 of the 5 cases with posterior semicircular canal dehiscence were associated to dehiscences in the superior canal. Saxby et al. [30] communicated 1 case of a child with bilateral superior unilateral and posterior canal dehiscence. Kundagari et al. [15] also presented a case of a patient with bilateral posterior semicircular canal dehiscence, together with right superior canal dehiscence. We found only 1 case in which the 2 dehiscences were associated, among the 61 patients studied, which denotes the low frequency of this association since they were selected patients.

The most frequent association found, which has clinical repercussion, was with the tegmen tympani. Crovetto et al. [5] found a $36.4 \%$ incidence of dehiscent tegmens associated with SSC dehiscence, and Nadaraja et al. [24] found $76 \%$. In the reverse study, El Hadi et al. [7] found a $56.5 \%$ incidence of dehiscent semicircular canals in patients who presented verified dehiscence of the tegmen tympani. We have observed this association in 28 of the cases studies (37.33\%) and we think that the explanation would be, as proposed by Fraile Rodrigo et al. [8], that both structures have a common origin, the otic capsule, and that the primary centres of the external or lateral SSCs, which, when they grow, extend to the base of the tegmental prolongation, collaborate in the ossification of the tegmen tympani. In addition to this, the external periostium layer, which separates the tegmen from the middle cranial fossa, is a continuation of the layer that covers the SSC. We have not found in the literature any manuscript that relates the posterior semicircular canal dehiscence with the absence of tegmen tympani, while we have found it in in 2 of the 4 cases of posterior semicircular canal dehiscence (50\%).
The association of SSC dehiscences with internal auditory canal dehiscences has only been described in literature by Manzari and Scagnelli [19]. We have observed 1 case in which SSC dehiscences were associated with internal auditory canal and posterior canal dehiscence.

In the literature consulted, we have not observed any association between SSC dehiscence and glenoid cavity dehiscence of the temporomandibular joint or of the tympanic bone, and only Kurt et al. [16] associates SSC dehiscence with symptoms in the temporomandibular joint, describing the flattening of the condyle, irregularities on the surface and formation of osteophytes, as radiological findings. We have seen these associations in a radiological study which leads us to consider that one same cause could act during development, both in the squamous portion of the temporal bone and in the otic capsule, although the origin is not yet clear.

Therefore, we have observed that the cases of SSC dehiscence associated with other temporal bone dehiscences are few and far between, either located in the petrous apophysis, in the squamous portion of the temporal bone or in the tympanic bone.

To understand the aetiology of multiple dehiscences, an embryological cause that acts upon the otic capsule must be sought. Thus, Isaacson and Vrabec [13] and Park et al. [26] observed that the bony otic capsule was significantly thinner in patients with associated SSC dehiscence. Gracia-Tello et al. [12] concludes that the existence of superior semicircular dehiscence is associated with the thinning of the bone of the canal on the opposite side, and Manzari and Modugno [18] think that the association between two bone structures that present dehiscences, and derive from the otic capsule, is presented as support to the idea that it is a congenital type disorder.

Niesten et al. [25] inform of three families in which the first-degree relatives show SSC dehiscences, and El Hadi et al. [7] registered two twins in the series that they studied, with both SSC dehiscence and tegmen tympani dehiscence. These events suggest genetic aetiology. In our study, we have found SSC dehiscence in 2 sisters, which suggests, in that case, a genetic component. However, in our studies about $\mathrm{COACH}$ syndrome and in the studies conducted by Roknic et al. [28], demonstrating a constant genetic disorder associated with these dehiscences has not been possible.

Wackym et al. [31] coins a new term, "otic capsule syndrome", to define the existence of associated de- 
hiscences between the different semicircular canals, and he bases this on the fact that these pathologies have very similar symptoms, whether implanted in (superior and posterior) vertical canals or in the (lateral) horizontal canal. We would use this term for when in the CT scans find multiple dehiscences of structures derived from the otic capsule (tegmen tympani, mastoid antrum, posterior semicircular conduct, internal auditory canal).

We have not found clinical features to distinguish patients with SSC dehiscence from the posterior, and both with other temporal bone dehiscences.

\section{CONCLUSIONS}

Therefore, and in conclusion, we think that SSC dehiscence may have a congenital cause and be associated with other dehiscences, and in our clinical practice, we must seek these associations whenever we encounter a disorder in the bony coverage of the temporal bone, as already recommended when a tegmen tympani or SSC dehiscence is diagnosed.

In agreement with our observations, reviews and after analysing the embryological development, we consider that the existence of malformation syndromes in which there is an association of multiple structure dehiscences, both of the otic capsule and of the temporal bone, is very likely.

\section{REFERENCES}

1. Allen $\mathrm{KP}$, Perez $\mathrm{CL}$, Isaacson $\mathrm{B}$, et al. Superior semicircular canal dehiscence in patients with spontaneous cerebrospinal fluid otorrhea. Otolaryngol Head Neck Surg. 2012; 147(6): 1120-1124, doi: 10.1177/0194599812457545, indexed in Pubmed: 22886079.

2. Bear ZW, McEvoy TP, Mikulec AA. Quantification of hearing loss in patients with posterior semicircular canal dehiscence. Acta Otolaryngol. 2015; 135(10): 974-977, doi: 10.3109/00016489.2015.1060630, indexed in Pubmed: 26107020.

3. Chen EY, Paladin A, Phillips G, et al. Semicircular canal dehiscence in the pediatric population. Int J Pediatr Otorhinolaryngol. 2009; 73(2): 321-327, doi: 10.1016/j. ijporl.2008.10.027, indexed in Pubmed: 19091428.

4. Cho YW, Shim BS, Kim JiW, et al. Prevalence of radiologic superior canal dehiscence in normal ears and ears with chronic otitis media. Laryngoscope. 2014; 124(3): 746-750, doi: 10.1002/lary.24281, indexed in Pubmed: 23794324

5. Crovetto M, Whyte J, Rodriguez OM, et al. Anatomo-radiological study of the superior semicircular canal dehiscence radiological considerations of superior and posterior semicircular canals. Eur J Radiol. 2010; 76(2): 167-172, doi: 10.1016/j.ejrad.2009.05.038, indexed in Pubmed: 19540691.
6. Dang PT, Kennedy TA, Gubbels SP. Simultaneous, unilateral plugging of superior and posterior semicircular canal dehiscences to treat debilitating hyperacusis. J Laryngol Otol. 2014; 128(2): 174-178, doi: 10.1017/ S0022215113003605, indexed in Pubmed: 24480590.

7. El Hadi T, Sorrentino T, Calmels MN, et al. Spontaneous tegmen defect and semicircular canal dehiscence: same etiopathogenic entity? Otol Neurotol. 2012; 33(4): 591-595, doi: 10.1097/MAO.0b013e31824bae10, indexed in Pubmed: 22429945.

8. Fraile Rodrigo JJ, Cisneros Al, Obón J, et al. Ontogenetic explanation for tegmen tympani dehiscence and superior semicircular canal dehiscence association. Acta Otorrinolaringol Esp. 2016; 67(4): 226-232, doi: 10.1016/j. otorri.2015.09.006, indexed in Pubmed: 26738982.

9. Gartrell BC, Gentry LR, Kennedy TA, et al. Radiographic features of superior semicircular canal dehiscence in the setting of chronic ear disease. Otol Neurotol. 2014; 35(1): 91-96, doi: 10.1097/MAO.0b013e3182a03522, indexed in Pubmed: 24136312.

10. Gianoli G. Deficiency of the superior semicircular canal. Curr Opin Otolaryngol Head Neck Surg. 2001; 9(5): 336-341, doi: 10.1097/00020840-200110000-00016.

11. Gopen Q, Zhou G, Poe D, et al. Posterior semicircular canal dehiscence: first reported case series. Otol Neurotol. 2010; 31(2): 339-344, doi: 10.1097/MAO.0b013e3181 be65a4, indexed in Pubmed: 19841602.

12. Gracia-Tello B, Cisneros A, Crovetto R, et al. Effect of semicircular canal dehiscence on contralateral canal bone thickness. Acta Otorrinolaringol Esp. 2013; 64(2): 97-101, doi: 10.1016/j.otorri.2012.10.004, indexed in Pubmed: 23298437.

13. Isaacson B, Vrabec JT. The radiographic prevalence of geniculate ganglion dehiscence in normal and congenitally thin temporal bones. Otol Neurotol. 2007; 28(1): 107-110, doi: 10.1097/01.mao.0000235968.53474.77, indexed in Pubmed: 17031323.

14. Krombach GA, DiMartino E, Schmitz-Rode T, et al. Posterior semicircular canal dehiscence: a morphologic cause of vertigo similar to superior semicircular canal dehiscence. Eur Radiol. 2003; 13(6): 1444-1450, doi: 10.1007/s00330003-1828-5, indexed in Pubmed: 12764665.

15. Kundaragi NG, Mudali S, Karpagam B, et al. Intracranially protruded bilateral posterior and superior SCCs with multiple dehiscences in a patient with positional vertigo: CT and MR imaging findings and review of literature. Indian J Radiol Imaging. 2014; 24(4): 406-409, doi: 10.4103/09713026.143904, indexed in Pubmed: 25489134.

16. Kurt H, Orhan K, Aksoy S, et al. Evaluation of the superior semicircular canal morphology using cone beam computed tomography: a possible correlation for temporomandibular joint symptoms. Oral Surg Oral Med Oral Pathol Oral Radiol. 2014; 117(3): e280-e288, doi: 10.1016/j. oooo.2014.01.011, indexed in Pubmed: 24528799.

17. Lookabaugh S, Kelly HR, Carter MS, et al. Radiologic classification of superior canal dehiscence: implications for surgical repair. Otol Neurotol. 2014; 36(1): 118-125, doi: 10.1097/ MAO.0000000000000523, indexed in Pubmed: 25122602.

18. Manzari L, Modugno GC. Bilateral dehiscence of both superior and posterior semicircular canals. Otol Neurotol. 2009; 
30(3): 423-425, doi: 10.1097/MAO.0b013e3181684048, indexed in Pubmed: 18997636.

19. Manzari L, Scagnelli P. Large bilateral internal auditory meatus associated with bilateral superior semicircular canal dehiscence. Ear Nose Throat J. 2013; 92(1): 25-33, doi: 10.1177/014556131309200109, indexed in Pubmed: 23354888.

20. Manzari L. Multiple dehiscences of bony labyrinthine capsule. A rare case report and review of the literature. Acta Otorhinolaryngol Ital. 2010; 30(6): 317-320, indexed in Pubmed: 21808455.

21. Meehan T, Nogueira C, Rajenderkumar D, et al. Dehiscence of the posterior and superior semicircular canal presenting in pregnancy. B-ENT. 2013; 9(2): 165-168, indexed in Pubmed: 23909125.

22. Minor LB, Solomon D, Zinreich JS, et al. Sound- and/ /or pressure-induced vertigo due to bone dehiscence of the superior semicircular canal. Arch Otolaryngol Head Neck Surg. 1998; 124(3): 249-258, doi: 10.1001/ archotol.124.3.249, indexed in Pubmed: 9525507.

23. Minor L. Superior canal dehiscence syndrome. Am J Otolaryngol. 2000; 21(1): 9-19, doi: 10.1016/s01960709(00)80068-x.

24. Nadaraja GS, Gurgel RK, Fischbein NJ, et al. Radiographic evaluation of the tegmen in patients with superior semicircular canal dehiscence. Otol Neurotol. 2012; 33(7): 1245-1250, doi: 10.1097/MAO.0b013e3182634e27, indexed in Pubmed: 22872173.

25. Niesten MEF, Lookabaugh S, Curtin $\mathrm{H}$, et al. Familial superior canal dehiscence syndrome. JAMA Otolaryngol Head Neck Surg. 2014; 140(4): 363-368, doi: 10.1001/ jamaoto.2013.6718, indexed in Pubmed: 24526223.
26. Park JH, Kang SIl, Choi HS, et al. Thickness of the bony otic capsule: etiopathogenetic perspectives on superior canal dehiscence syndrome. Audiol Neurootol. 2015; 20(4): 243-250, doi: 10.1159/000371810, indexed in Pubmed: 25998441.

27. Puram SV, Roberts DS, Niesten MEF, et al. Cochlear implant outcomes in patients with superior canal dehiscence. Cochlear Implants Int. 2015; 16(4): 213-221, doi: 10.1179/1754762813Y.0000000044, indexed in Pubmed: 24074366.

28. Roknic N, Huber A, Hegemann SCA, et al. Mutation analysis of Netrin 1 and HMX3 genes in patients with superior semicircular canal dehiscence syndrome. Acta Otolaryngol. 2012; 132(10): 1061-1065, doi: 10.3109/00016489.2012.681797, indexed in Pubmed: 22779713.

29. Russo JE, Crowson MG, DeAngelo EJ, et al. Posterior semicircular canal dehiscence: CT prevalence and clinical symptoms. Otol Neurotol. 2014; 35(2): 310-314, doi: 10.1097/MAO.0000000000000183, indexed in Pubmed: 24366470.

30. Saxby AJ, Gowdy C, Fandiño M, et al. Radiological prevalence of superior and posterior semicircular canal dehiscence in children. Int J Pediatr Otorhinolaryngol. 2015; 79(3): 411-418, doi: 10.1016/j.ijporl.2015.01.001, indexed in Pubmed: 25636666.

31. Wackym PA, Wood SJ, Siker DA, et al. Otic capsule dehiscence syndrome: Superior semicircular canal dehiscence syndrome with no radiographically visible dehiscence. Ear Nose Throat J. 2015; 94(8): E8-EE24, doi: 10.1177/014556131509400802, indexed in Pubmed: 26322461 